OPEN ACCESS

Edited by:

Andrew Philp,

Garvan Institute of Medical

Research, Australia

Reviewed by:

Kunihiro Sakuma,

Tokyo Institute of Technology, Japan

Craig Andrew Goodman,

Victoria University, Australia

*Correspondence:

Katrien De Bock

katrien-debock@ethz.ch

Specialty section:

This article was submitted to

Striated Muscle Physiology,

a section of the journal

Frontiers in Physiology

Received: 01 July 2019 Accepted: 19 September 2019

Published: 04 October 2019

Citation:

D'Hulst G, Palmer AS, Masschelein E, Bar-Nur O and De Bock K (2019)

Voluntary Resistance Running as a Model to Induce mTOR Activation in Mouse Skeletal Muscle.

Front. Physiol. 10:1271.

doi: 10.3389/fphys.2019.01271

\section{Voluntary Resistance Running as a Model to Induce mTOR Activation in Mouse Skeletal Muscle}

\author{
Gommaar D'Hulst', Andrew S. Palmer ${ }^{2}$, Evi Masschelein ${ }^{1}$, Ori Bar-Nur ${ }^{2}$ and Katrien De Bock ${ }^{\text {1* }}$ \\ 'Laboratory of Exercise and Health, Department of Health Sciences and Technology, Swiss Federal Institute of Technology \\ (ETH) Zurich, Zurich, Switzerland, '2Laboratory of Regenerative and Movement Biology, Department of Health Sciences and \\ Technology, Swiss Federal Institute of Technology (ETH) Zurich, Zurich, Switzerland
}

Long-term voluntary resistance running has been shown to be a valid model to induce muscle growth in rodents. Moreover, the mammalian target of rapamycin complex 1 (mTORC1) is a key signaling complex regulating exercise/nutrient-induced alterations in muscle protein synthesis. How acute resistance running affects mTORC1 signaling in muscle and if resistance applied to the wheel can modulate mTORC1 activation has not yet been fully elucidated. Here, we show that both acute resistance running and acute free running activated $\mathrm{mTORC} 1$ signaling in the $m$. gastrocnemius, $m$. soleus, and $m$. plantaris, but not in $m$. tibialis anterior of mice when compared to sedentary controls. Furthermore, only the low threshold oxidative part in the $\mathrm{m}$. gastrocnemius showed increased mTORC1 signaling upon running and acute heavy-load resistance running evoked higher downstream mTORC1 signaling in both $\mathrm{m}$. soleus and $\mathrm{m}$. plantaris than free running without resistance, pointing toward mechanical load as an important independent regulator of mTORC1. Collectively, in this study, we show that voluntary resistance running is an easy-to-use, time-efficient and low stress model to study acute alterations in mTORC1 signaling upon high-load muscular contractions in mice.

Keywords: mTORC1, signaling, resistance running, load, acute

\section{INTRODUCTION}

Maintaining skeletal muscle mass throughout life is critical as loss of muscle mass is associated with increased mortality (Szulc et al., 2010), higher disability, loss of function (Janssen et al., 2002), and increased risk of falls (Szulc et al., 2004). Resistance exercise increases muscle mass (Bhasin et al., 1996; Abe et al., 2003; Verdijk et al., 2009) and improves strength outcomes (Pyka et al., 1994; Staron et al., 1994), but the mechanisms by which high load contractions regulate skeletal muscle fiber size are incompletely understood. In an attempt to elucidate these mechanisms, many resistance-based exercise models in mice have been developed (Cholewa et al., 2014), such as synergistic ablation (Goldberg, 1968), electrical stimulation (Baar and Esser, 1999), and chronic stretch (Goldspink, 1999). Although all valuable, they are invasive, cumbersome, and often do not mimic real life scenarios. Remarkably, many mouse strains voluntarily run large distances when given access to a running wheel (De Bono et al., 2005). Moreover, external resistance can be added to the wheel to increase muscle force production (Soffe et al., 2016). Therefore, as mouse handling and stress is minimal, the running wheel is considered an excellent model of physiological training with similar muscular adaptations as other well-accepted models 
such as synergistic ablation and electrical stimulation. Indeed, many groups have shown that voluntary resistance running leads to muscular hypertrophy in rodents (Legerlotz et al., 2008; Call et al., 2010; White et al., 2016; Mobley et al., 2018).

mTOR is a protein complex, which acts as a conductor in cell growth (Wolfson and Sabatini, 2017). In particular, mTOR complex 1 (mTORC1) integrates nutrient, growth, and stress signals to promote protein synthesis (Goodman et al., 2011; Huang and Fingar, 2014). Activation of mTOR is known to increase ribosomal biogenesis and protein translation (Mahajan, 1994; Thoreen et al., 2012; Figueiredo et al., 2015), two processes, which play a central role in the adaptation to resistance training or chronic overload (Bodine et al., 2001; Drummond et al., 2009; Ogasawara and Suginohara, 2018). Both a single bout or repeated bouts of resistance exercise can increase mTOR signaling in muscle (Baar and Esser, 1999; Kubica et al., 2005; Burd et al., 2010; Dreyer et al., 2010) and an enhancement in mTOR activation after resistance exercise is related to increased muscle mass (Baar and Esser, 1999). To confirm the role of mTOR on muscle hypertrophy, blocking mTOR signaling with compounds such as rapamycin or AZD8055 has been demonstrated to reduce protein synthesis and muscle growth (Bodine et al., 2001; Drummond et al., 2009; Ogasawara and Suginohara, 2018), underscoring the indispensable role of mTORC1 in muscular adaptations to resistance type exercise. Data on voluntary resistance running and mTOR activation are sparse, but one study showed no increase in mTOR activation after 8 months of resistance running in the m. quadriceps, despite hypertrophy in most of the hindlimb muscles, including the m. quadriceps (White et al., 2016). Interpretation of this data might be difficult since the mice were aged, samples were harvested several hours after exercise, no standardization of food intake before sample harvesting occurred, and - consistent with human data (Brook et al., 2015) - resistance training could have resulted in an attenuated activation of mTORC1 in response to resistance training. Additionally, other hindlimb muscles were not examined for downstream mTORC1 signaling (White et al., 2016). Thus, to date, the mechanisms behind resistance running-induced muscle hypertrophy are unclear.

Therefore, the aim of this study was to explore whether voluntary resistance running can be used as a non-invasive stress-free model to study and acutely modulate mTORC1 signaling in vivo.

\section{MATERIALS AND METHODS}

\section{Animals}

All experiments were performed on male C57BL/6 J mice. All mice used for the experiments were housed in individually ventilated cages (3-4 littermates per cage) at standard housing conditions $\left(22^{\circ} \mathrm{C}, 12 \mathrm{~h}\right.$ light/dark cycle, dark phase starting at $7 \mathrm{pm}$ ), with ad libitum access to chow (KlibaNafag, diet \#3436 and diet \#3437) and water. Health status of all mouse lines was regularly monitored according to FELASA guidelines.

\section{Experimental Procedures}

All animal procedures were approved by the Veterinary office of the Canton of Zürich (license nr. ZH254-16). During the intervention, mice were individually housed in open cages equipped with a running wheel device (TSE Systems). The running wheel device continuously records wheel movements out of which total distance $(\mathrm{km})$, speed $\left(\mathrm{m} . \mathrm{s}^{-1}\right)$, number of running bouts, and resistance on the wheel $(\mathrm{N})$ can be extracted. Additionally, to increase the force needed to rotate the wheel, resistance $(0-100 \%)$ can be added. To calculate total work, we used $W=P t$, where $W$ is work, $P$ is power, and $t$ is time. To calculate the power of the wheel at each braking resistance, we used the equation $P=2 \pi \times f \times M$, where $f$ is the angular frequency of the wheel, and $M$ is the torque at a given braking resistance. TSE Systems provided a torque braking resistance curve for which a braking resistance of $60 \%$ has a constant torque of 0.0125 N.m.

\section{Experiment 1}

An overview of the experimental procedures can be found in Figure 1. About 14 to 16-week-old male C57BL/6 J mice were randomly assigned to either the voluntary resistance-running group (VResRun) or sedentary (Sed) control group. VResRun mice were familiarized to the resistance running protocol for 1 week. During familiarization, VResRun mice had access to the wheel; with $0 \%$ braking resistance on the first night, with $20 \%$ braking resistance on the fourth night, and with $40 \%$ braking resistance on the seventh night. On the second, third, fifth, and sixth night, the running wheels were blocked. After the seventh night, mice remained in the resistance wheel cages without access to the running wheel for four nights to mitigate any potential

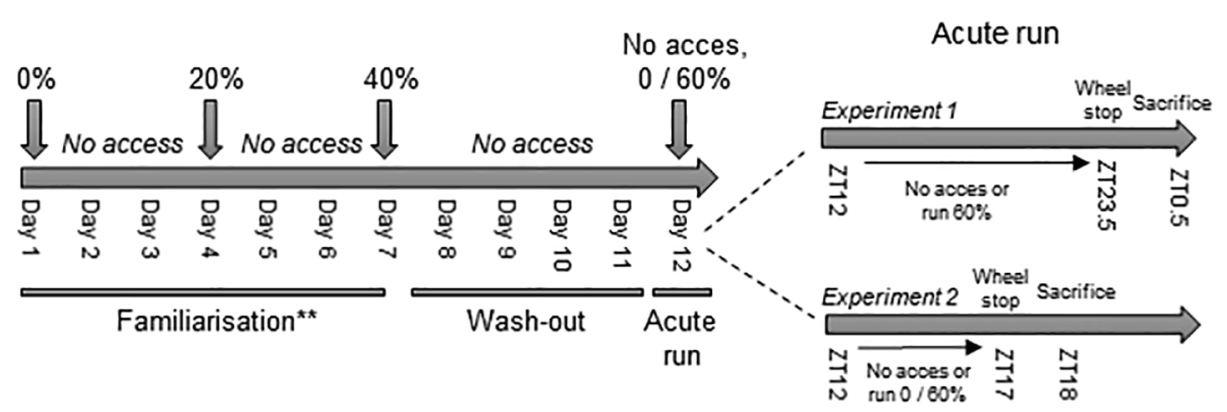

FIGURE 1 | Experimental set-up. ${ }^{* \star}$ In experiment 1, only the VResRun group was familiarized to the wheel. The Sed remained sedentary during the whole experiment. In experiment 2, all groups were familiarized to the wheel, including the Sed. 
training effects. On the twelfth and final day of the intervention, mice had access to the running wheel at $60 \%$ braking resistance for the 12-h dark cycle (ZT12-ZT23.5). Sedentary mice did not undergo familiarization and were housed in a resistance running wheel cage with the wheel blocked. At ZT23.5, running wheels were blocked, mice were removed from running cages and fasted for $1 \mathrm{~h}$. Muscle samples were collected $1 \mathrm{~h}$ after cessation of running, since at this time point, eccentric contraction induced activation of mTORC1 is maximal (O’Neil et al., 2009).

\section{Experiment 2}

Around 10-16-week-old male C57BL/6 mice were randomly allocated either to a Sed, voluntary run (VRun) or VResRun group. This time, Sed, VRun, and VResRun groups were all familiarized following the aforementioned familiarization protocol (Figure 1). Access to the running wheels was blocked for four nights. On the final night, VResRun mice ran at $60 \%$ braking resistance, Run mice ran at $0 \%$ braking resistance from ZT12 to ZT17 and Sed did not run (wheel blocked). Mice were removed from running cages at ZT17 am and fasted for $1 \mathrm{~h}$ prior to sample collection.

\section{Sample Collection}

Mice were fasted for $1 \mathrm{~h}$ at ZT23.5 (experiment 1) and ZT17 (experiment 2) and anesthetized using Ketamine/Xylazine $10 \mu \mathrm{g} \cdot \mathrm{g}^{-1}$ and acepromazine $\left(2-5 \mathrm{mg} \cdot \mathrm{kg}^{-1}\right)$ body weight via intraperitoneal injection. The depth of anesthesia was confirmed by testing pedal withdrawal reflex before tissue collection. Subsequently, the $m$. gastrocnemius (GAS), $m$. tibialis anterior (TA), $m$. soleus $(S O L), m$. plantaris (PLT) and $m$. triceps (TRI) were dissected and snap frozen. One GAS was frozen in OCT embedding matrix (CellPath) in 2-Methylbutane (sigma Aldrich) on liquid nitrogen for histochemical analysis. After sample collection, animals were euthanized and major bleeding was induced to confirm death.

\section{Protein Extraction and Western Blot Sample Preparation}

Between 10 and $25 \mathrm{mg}$ of muscle sample was homogenized in ice cold lysis buffer (1:10, w/v) (50 mM Tris- $\mathrm{HCl} \mathrm{pH} 7.0$, $270 \mathrm{mM}$ sucrose, $5 \mathrm{mM}$ EGTA, $1 \mathrm{mM}$ EDTA, $1 \mathrm{mM}$ sodium orthovanadate, $50 \mathrm{mM}$ glycerophosphate, $5 \mathrm{mM}$ sodium pyrophosphate, $50 \mathrm{mM}$ sodium fluoride, $1 \mathrm{mM} \mathrm{DTT,} 0.1 \%$ Triton-X 100 and $10 \%$ protease inhibitor) (20 $\mu$ l per $1.8-2.5$ of tissue sample) using an OMNI-THq Tissue homogenizer (OMNI International) for $20 \mathrm{~s}$ until a consistent homogenate was formed. Samples were centrifuged at $4^{\circ} \mathrm{C}$ at $10,000 \mathrm{~g}$ for $10 \mathrm{~min}$ and the supernatant with proteins collected. Protein concentration was determined using the DC assay protein method (Biorad Laboratories) to equalize the amount of protein.

\section{Protein Transfer}

Samples were prepared 3:4 with laemmli buffer containing 10\% 2-mercaptoethanol (Bio-rad laboratories) and heated at $95^{\circ}$ for 5 min. Proteins were run on a $4-20 \%$ Mini-PROTEAN TGX Stain Free Pre-Cast Gel (Biorad Laboratories) for $45 \mathrm{~min}$ at 120-140 V and subsequently transferred onto immuno-Blot PVDF
Membranes (Biorad Laboratories) at $90 \mathrm{~V}$ for $100 \mathrm{~min}$. Membranes were cut according to desired proteins, blocked for $1 \mathrm{~h}$ at room temperature in 5\% milk in TBS-T, TBS (1:10, w/v) (24.23 g Trizma $\mathrm{HCl}, 80.06 \mathrm{~g} \mathrm{NaCl}$ in $800 \mathrm{ml}$ ultra-pure water $\mathrm{pH}$ 7.6, topped up to $1 \mathrm{~L}$ ) with $1 \mathrm{ml}$ of Tween, and incubated overnight with the following primary antibodies 1:1,000 (Cell Signaling); $\mathrm{pS6K}^{\text {Thr389 }}$ (\#9206), pS6K1 ${ }^{\text {Thr421/Ser424 }}$ (\#9204), pRPS6 ${ }^{\text {Ser235/236 }}$ (\#2211), pSMAD2 $2^{\text {Ser245/250/255 }}$ (\#3104), p4E-BP1Ser ${ }^{65}$ (\#9451), and pSAPK/ $\mathrm{JNK}^{\text {Thr183/Tyr185 }}$ (\#9251). The membranes were washed for $10 \mathrm{~min}$ three times in TBS-T and subsequently incubated in secondary antibody 1:5,000 in TBS-T with 5\% milk (Anti-rabbit IgG, HRP-linked Antibody \#7074, Cell Signaling) for $1 \mathrm{~h}$ at room temperature. Proteins were washed for $10 \mathrm{~min}$ three times in TBS-T and incubated for $30 \mathrm{~s}$ in 1:1 Luminol/Enhancer solution and peroxide solution. Membranes were imaged with a Biorad Chemidoc Touch Imaging System (Biorad Laboratories).

\section{Image Analysis}

Images were quantified using Image Lab software (Biorad Laboratories) using the volume tools function with each band and lane quantified with the same volume or area. Total membrane protein or total gel loading was used as a loading control (Rivero-Gutiérrez et al., 2014) and a positive control sample was loaded to compare across gels. All quantified volumes were divided by the positive control and normalized to the control group.

\section{Immunohistochemistry Sample Preparation P-RPS6}

About $10 \mu \mathrm{m}$ sections of muscle embedded in OCT were made using a cryostat (Leica CM 1950) and collected on Superfrost Ultra Plus slides (Thermo Scientific). Muscle samples were fixed with $-20^{\circ} \mathrm{C}$ acetone for $10 \mathrm{~min}$ and subsequently incubated in PBS for $15 \mathrm{~min}$. Slides were blocked for $1 \mathrm{~h}$ at room temperature in solution A (PBS with 5\% normal goat serum and $0.3 \%$ CHAPS) and then incubated overnight at $4^{\circ} \mathrm{C}$ in solution $\mathrm{B}$ (PBS with $0.5 \%$ BSA and $0.3 \%$ CHAPS) containing primary antibody p-RPS6 $6^{\text {Ser235/236 }}$ rabbit conjugated (1:200, cell signaling). The next day samples were washed three times with PBS and then incubated for $1 \mathrm{~h}$ at room temperature in solution $\mathrm{A}$ containing secondary antibodies Goat-anti rabbit IgG Alexa Fluor 488 (1:250, Invitrogen). Slides were once again washed two times for $5 \mathrm{~min}$ in PBS and then washed for $10 \mathrm{~min}$ with PBS containing WGA alexa fluor 647 (1:400, Invitrogen). Slides were mounted with immuno-mount (Thermo Scientific) and a glass coverslip and allowed to dry. A sample that followed the above procedure but was not incubated with primary antibodies was used as a negative control. Images were taken using an epifluorescence microscope (Zeiss Axio observer Z.1) at 20x using Zen Pro software. An image of a sedentary sample was used to define imaging settings and applied to all subsequent images.

\section{Fiber Typing}

About $10 \mu \mathrm{m}$ sections were dried and washed for $5 \mathrm{~min}$ in PBS supplemented with $0.05 \%$ triton (PBST) and subsequently blocked for $60 \mathrm{~min}$ in PBST $+10 \%$ goat serum (ThermoFisher 
Scientific, 16200-064). Afterward, a primary antibody cocktail was applied for $120 \mathrm{~min}$ for myosin heavy chain I (1/50), IIa (1:200) (Developmental studies hybridoma bank) diluted in PBST $+10 \%$ goat serum. After washing three times for $5 \mathrm{~min}$, a secondary antibody cocktail, diluted in PBST $+10 \%$ goat serum, was applied for goat anti-mouse Alexa Fluor 488, 350 and wheat germ agglutinin Alexa fluor 647 (1:250) for $60 \mathrm{~min}$. Slides were mounted after a $3 \times 5$ min wash, sealed with glass cover slips and imaged with a epifluorescent microscope (Zeiss Axio observer Z.1) at $10 \times$.

\section{Statistical and Data Analyses}

Results are presented as mean with standard error of the mean (SEM) bars and individual data points. An unpaired two-tailed Student's $t$-test was used for generating a $p$ in experiment 1 when comparing two groups. Data in experiment 2 were subjected to a one-way analysis of variance (ANOVA) to generate a $p$ value and post hoc tests were performed using Tukey's post hoc test using Graphpad Prism to compare between groups. Significance was set at $p<0.05$.

\section{RESULTS}

\section{Acute Resistance Running Activates the mTOR Signaling Pathway in Mouse Skeletal Muscle}

To determine if resistance exercise activates mTOR signaling within skeletal muscle, we measured the phosphorylation of downstream mTORC1 target kinases $1 \mathrm{~h}$ after cessation of one night of resistance running. In mice, phosphorylation levels of $\mathrm{pS} 6 \mathrm{~K} 1^{\text {Thr389 }}, \mathrm{pS} 6 \mathrm{~K} 1^{\text {Thr421/Ser424 }}, \mathrm{pRPS}^{\text {ser235/236 }}$, and $\mathrm{p} 4 \mathrm{EBP} 1^{\text {ser65 }}$ were highly increased in SOL of VResRun compared to SED mice (Figures 2A,C). Furthermore, VResRun also lead to a strong increase $\mathrm{pS} 6 \mathrm{~K} 1^{\text {Thr389 }}$ in PLT and GAS (Figure 2B). Interestingly, there were no significant differences in phosphorylation of any mTOR targets in the TA muscle (Figure 2B) or TRI (data not shown). This data shows that VResRun activates downstream mTORC1 signaling.

The running activity over the night followed the pattern seen in Figure 3. Mice ran consistently and constantly between ZT12 and ZT18, after which they rested before recommencing running after ZT22. Interestingly, some mice did not restart at ZT22 for unknown reasons, a feature that could potentially induce variability in the signaling data. We therefore decided to further standardize the protocol by blocking the wheel at ZT17 and excise the muscles at ZT18 in experiment 2.

\section{Acute Resistance Running Activates Mammalian Target of Rapamycin Complex 1 More Than Non-resistance Running}

For the second experiment, we used the optimized protocol, and included an additional condition where no resistance was applied on the wheel (VRun). This would allow us to assess whether the observed increase in mTORC1 activity was caused by the increase in resistance applied on the wheel, rather than by running itself. Parameters from the free running and the resistance running bout are presented in Table 1. VResRun had to overcome 24 times more force per rotation of the wheel than the VRun, leading to 29 times more total work over the course

\section{A}

C

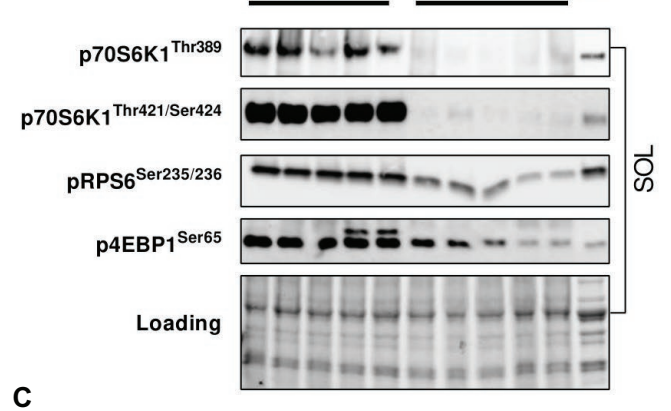

B

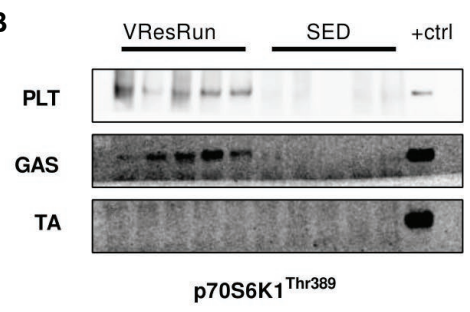

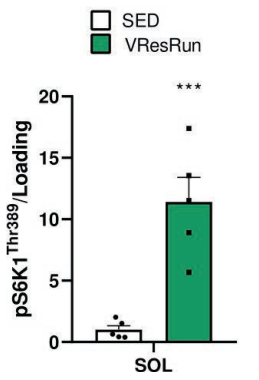
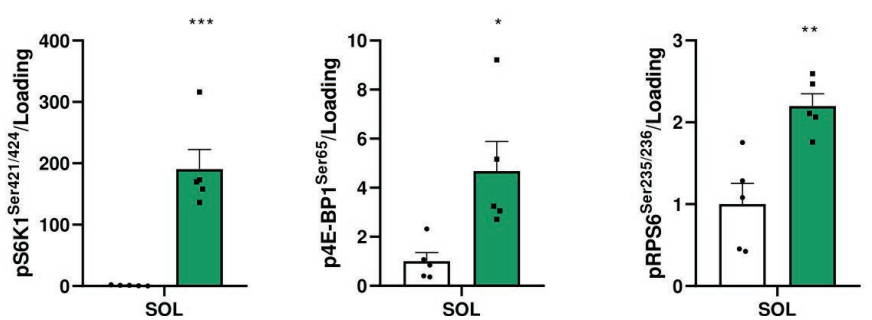

FIGURE 2 | The effect of one night of voluntary resistance running (VResRun, $n=5$ ) on mTORC1 downstream signaling. (A) Representative blots of pS6K1 $1^{\text {Thr389 }}$, pS6K1 $1^{\text {Thra2 } 1 / \text { Sert24 }}$, pRPS6 ${ }^{\text {Ser256/236 }}$, p4EBP1 ${ }^{\text {Ser65 }}$ in SOL. (B) Representative blots of pS6K1 $1^{\text {Thr389 }}$ in PLT, GAS, and TA. (C) Quantifications. (Sed; $n=5$ ). ${ }^{*} p<0.05$ vs. SED, ${ }^{\star *} p<0.01$ vs. SED, ${ }^{* \star} p<0.001$ vs. SED. 


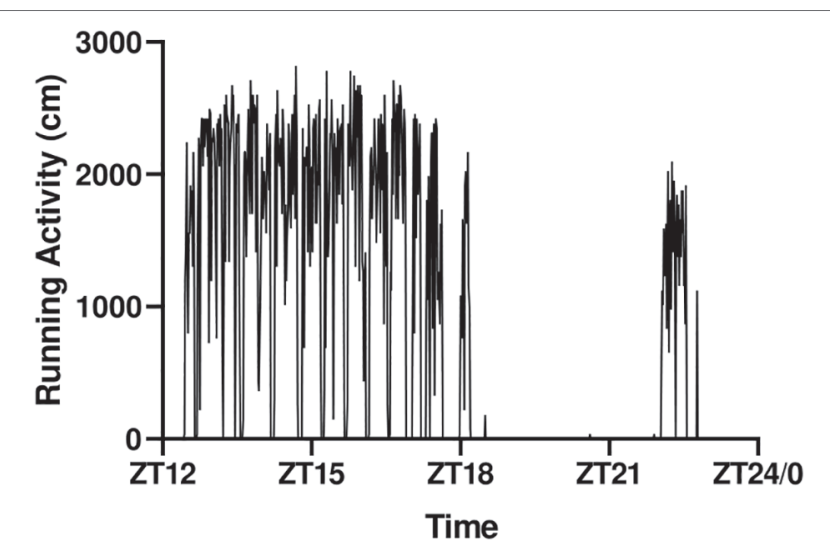

FIGURE 3 | Representative running pattern of a mouse during the active phase.

TABLE 1 | Running summary VRun vs. VResRun.

\begin{tabular}{lcc}
\hline & VRun & VResRun \\
\hline Distance $(\mathrm{km})$ & $3.46 \pm 0.95$ & $4.00 \pm 0.40$ \\
Time (min) & $129 \pm 31$ & $151 \pm 12$ \\
Sum of all runs & $1,014 \pm 272$ & $876 \pm 278$ \\
Speed (m.s $\left.{ }^{-1}\right)$ & $0.44 \pm 0.05$ & $0.44 \pm 0.02$ \\
Work $(\mathrm{J})$ & $30 \pm 8$ & $867 \pm 88^{\star \star \star}$ \\
Force $(\mathrm{N})$ & 0.009 & $0.22^{\star \star \star}$ \\
\hline
\end{tabular}

***Indicates $p<0.001$ vs. VRun. VRun, $n=6$; VResRun, $n=7$. of the $5 \mathrm{~h}$ run time-window $(p<0.001$, Table 1$)$. Both acute VRun and VResRun increased downstream mTORC1 signaling

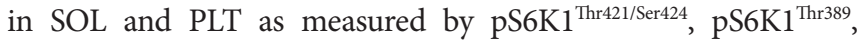
and pRPS6 $6^{\text {ser235/236 }}$ (Figures 4A-E). Notably, VResRun augmented downstream mTORC1 signaling significantly more than VRun both in the SOL and PLT, indicating that load independently augments mTORC1 signaling in an in vivo setting (Figures 4A-E). Finally, total work was highly correlated with pS6K1 $1^{\text {Thr421/Ser } 224}$ in both SOL $(r=0.69, p<0.05)$ and PLT $(r=0.63, p<0.05)$.

One of the proposed pathways of how high-load contractions regulate mTORC1 is via activation of the stress responsive mitogen-activated protein kinase (MAPK) pathway (Rahnert and Burkholder, 2013). More specifically, c-Jun N-terminal kinase (JNK), a member of the MAPK family, has been put forward as the molecular switch that, when active, promotes muscle hypertrophy and, when inhibited, allows endurance training adaptations such as enhanced aerobic capacity (Lessard et al., 2018). To investigate whether the increased mTORC1 signaling in VResRun muscle was related to increased JNK activation when compared to predominantly aerobic (VRun) or sedentary (SED) muscle, we measured pSAPK/JNK at Thr183/Tyr185 and its downstream target pSMAD2 at Ser245/250/255. VResRun increased pJNK and pSMAD2 modestly $(\sim 4$ fold, $p<0.05)$ in SOL only, while PLT showed higher variation and was unaffected (Figures 4A,B,F,G). Thus, while we observed increased mTORC1 activation upon VRun, this seemed not to be dependent on increased JNK and SMAD2 phosphorylation (Figures 4A,B,F,G).
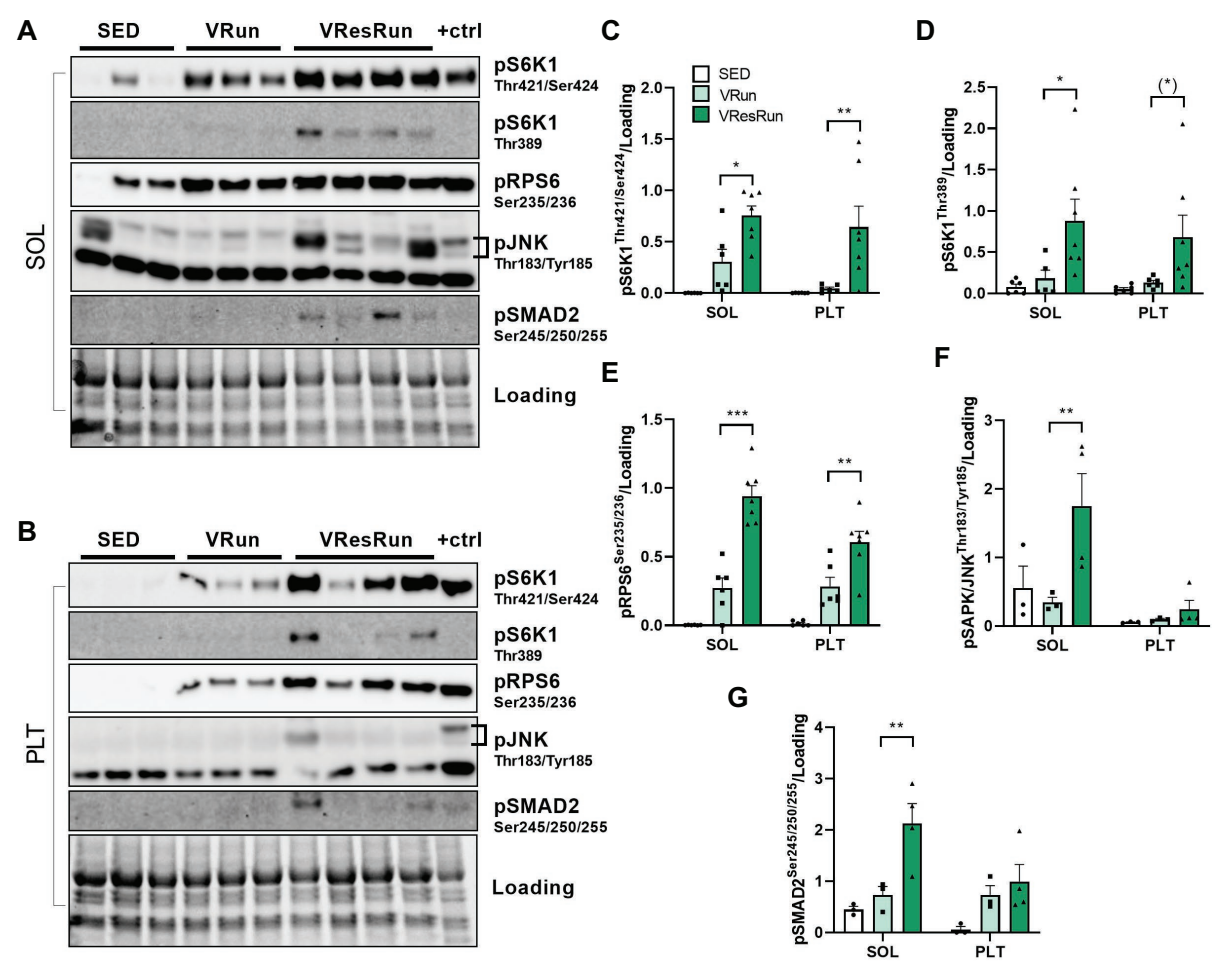

FIGURE 4 | The effect of $5 \mathrm{~h}$ of voluntary running (VRun, $n=6$ ) and voluntary resistance running (VResRun, $n=7$ ) on mTORC1 downstream signaling. (A) Representative blots in SOL. (B) Representative blots in PLT. (C) Quantification of pS6K1 ${ }^{\text {Thr421/Sert24. }}$. (D) Quantification of pS6K1 $1^{\text {Thr389 }}$. (E) Quantification of pRPS6 ${ }^{\text {Ser235/236. }}$. (F) Quantification

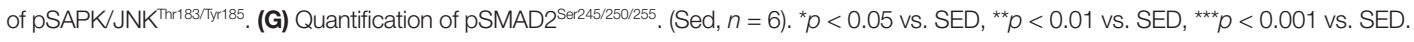




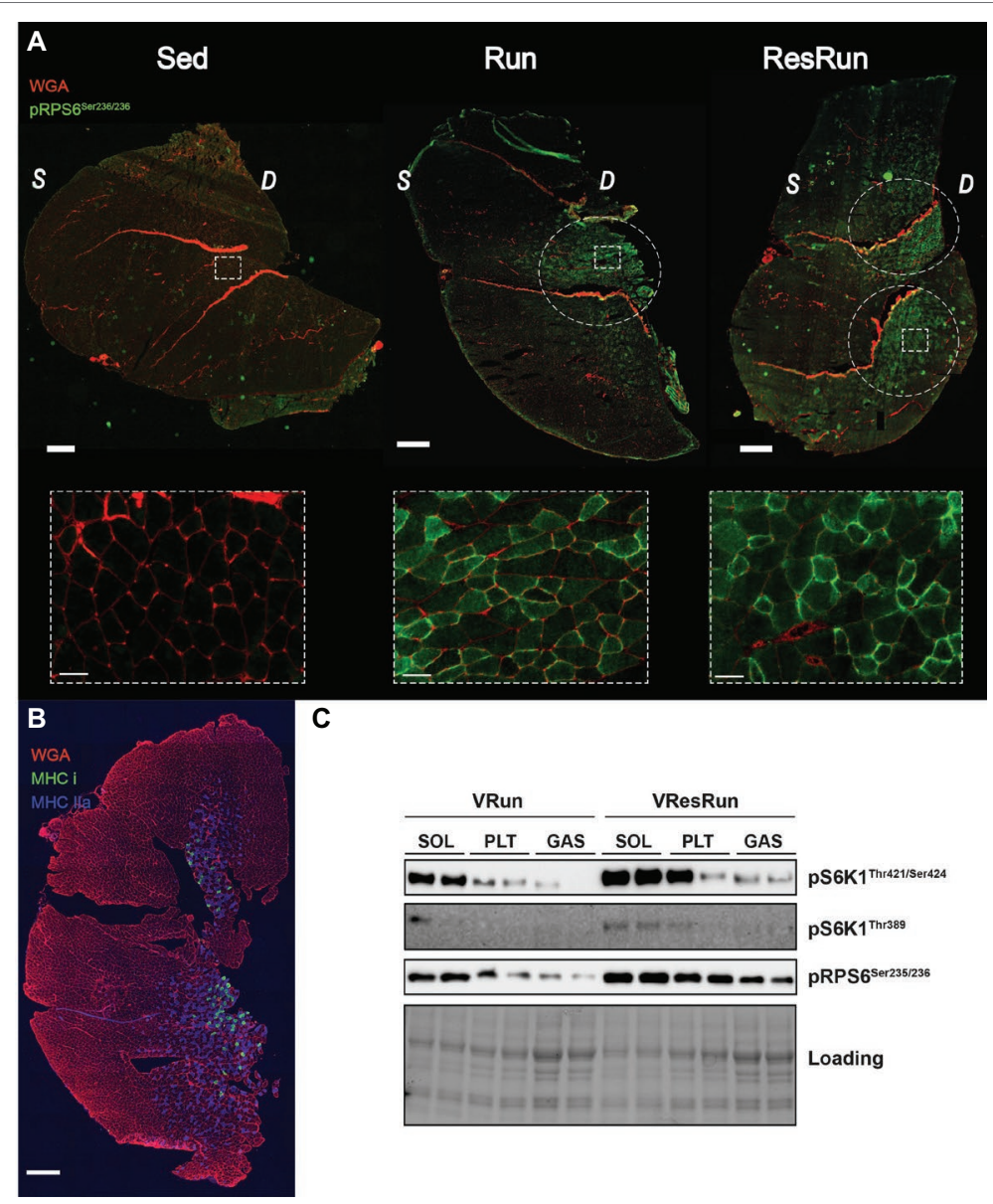

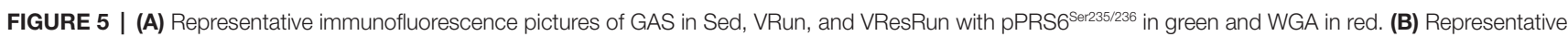
immunofluorescence staining of GAS with Myosin Heavy Chain type I in green, Myosin Heavy Chain type lla in blue and Wheat Germ Agglutinin WGA in red. (C) Representative blots of downstream mTORC1 signaling (VRun and VResRun) in SOL, PLT, and GAS. Scale bar in tile scan represents 500 mm and scale bar in zoom-in represents $50 \mu \mathrm{m}$; WGA, wheat germ agglutinin; $S$, superficial part; $D$, deep part.

To visualize activation and distribution of mTORC1 in mouse skeletal muscle, we performed an immunohistochemical staining

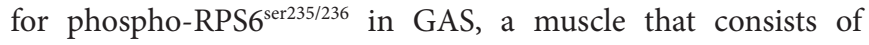
both oxidative and glycolytic fibers, organized in specific regions. Interestingly, only a selected amount of fibers were phosphorylated in the VRun and VResRun (Figure 5A) and they were exclusively situated in the oxidative part (on the anterior side) of the GAS (Figure 5B; Sher and Cardasis, 1976). This also represented itself in a lesser activation of downstream mTORC1 after acute running when the phosphorylation of MTORC1 was measured in the whole GAS compared to the SOL and PLT (Figure 5C). These data suggest that voluntary running activates downstream mTORC1 signaling in a select amount of predominantly oxidative fibers.

\section{DISCUSSION}

This study aimed to validate acute resistance running as a model of resistance exercise-induced mTORC1 activation in mouse skeletal muscle. Previous research has shown muscle hypertrophy (Legerlotz et al., 2008; White et al., 2016) and increased strength (Roemers et al., 2017) following voluntary resistance running, and we aimed to link these models with a key molecular pathway, which regulates muscle protein synthesis. We show increased mTORC1 activity in the PLT, SOL, and GAS, whereas we did not detect enhanced activation in the TA or TRI muscle, suggesting muscle specific differences in mTORC1 activation upon acute resistance running, which might be determined by specific load applied on these muscles during running. To further study the involvement of mechanical load, we subjected mice to voluntary free and resistance running and found increased downstream mTORC1 signaling in SOL and PLT when resistance was applied to the wheel.

Numerous studies showed increased muscle mass after resistance running in mice. It has been reported that muscle mass is increased in the PLT (Legerlotz et al., 2008; Holland et al., 2016; Mobley et al., 2018), SOL (Konhilas et al., 2005; Legerlotz et al., 2008; Call et al., 2010; White et al., 2016), 
GAS (Mobley et al., 2018), m. quadriceps (Soffe et al., 2016), and TRI (Call et al., 2010) after long term (>4 weeks) resistance running. Our findings provide evidence of a potential molecular basis for the above findings as we report increased mTORC1 activation after one night of resistance running in the aforementioned muscles. It has been reported before that during wheel running, the force shifts to the hindlimb (0.85:0.15) compared to overground running leading to a subsequent increase in hindlimb normal forces (Roach et al., 2012). It is therefore likely that the activation of mTORC1 after resistance running was load-dependent, as we did not find any, or minimal phosphorylation of its downstream kinases in the TRI of the forelimb. In agreement with this, we also failed to observe significant mTORC1 activation in TA, a dorsiflexor muscle that does not bear body weight, can produce less force than the plantar flexors (Ashton-Miller et al., 1992) and thus is likely recruited to a lower extent during (resistance) running when compared to muscles of the $m$. triceps surae.

At $60 \%$ resistance, the resistance we opted to put on the wheel in this study, the additional force needed to overcome the wheel resistance was $0.2 \mathrm{~N}$. Plantar flexion strength of the $m$. triceps surae is much higher [3.3 N (Ashton-Miller et al., 1992)] indicating that maximal force production was not required to overcome the resistance imposed by the wheel. Thus, during a single contraction only a subset of motor units, likely the oxidative fibers which are recruited during submaximal force production (Henneman et al., 1965), needed to be activated. This was underscored by our observations that in the GAS, only the areas containing more oxidative fibers (the anterior part close to the bone) were highly positive for pRPS6, while the glycolytic part remained unaffected after VResRun and VRun. These findings are corroborated by the fact that nearly all aforementioned studies investigating hypertrophy after wheel running found significant hypertrophy in the SOL and/or PLT, while GAS and dorsiflexors were much less, or even not at all affected (Legerlotz et al., 2008; White et al., 2016). Nevertheless, future research needs to further evaluate whether load-dependent mTORC1-signaling directly affects hypertrophy in a musclespecific way.

Besides to a large increase in downstream mTORC1 signaling after voluntary resistance running, we also observed a significant, albeit $~ 50 \%$ less potent increase in pS6K1 and pRPS6 in mice that acutely ran for a night without any resistance on the wheel. To the best of our knowledge, this is the first study that specifically alters load in an acute in vivo physiological training setting to modulate intra-muscular downstream mTORC1 signaling. The increase in mTORC1 activity after acute endurance exercise (VRun) was somewhat surprising as previous data has reported a decrease in mTORC1 activation $0.5,3$ and $6 \mathrm{~h}$ after a single bout of uphill treadmill running (18 $\mathrm{m} \cdot \mathrm{min}^{-1}$ for $1 \mathrm{~h}$ at a $5^{\circ}$ gradient) (Philp et al., 2015). On the other hand, studies using longer duration (and intensity) of running did indeed find higher downstream mTORC1 signaling immediately and $3 \mathrm{~h}$ after the running bout (Scribbans et al., 2014). In an attempt to elucidate the mechanisms that regulate muscular plasticity, a recent report showed JNK/
SMAD2 to act as a major switch between muscle growth and endurance phenotype when the kinases were activated or inactivated, respectively (Lessard et al., 2018). We report an increase in pJNK1/2 and pSMAD2 in VResRun, while this effect was absent in the VRun condition. The absence of JNK activation and the induction of mTORC1 after VRun suggest that VRun is indeed a good model for endurance adaptations in skeletal muscle and that mechanisms other than the mechanosensitive MAPK signaling regulate mTORC1 upon endurance type training.

To conclude, we show that acute resistance running and acute free running increased activation of the MTORC1 pathway in mouse muscle. Our data suggest that this activation is dependent on load as the $m$. triceps surae muscles had higher activation compared to the hindlimb dorsi flexors and only a subset of oxidative fibers were activated in the GAS. Furthermore, resistance running, in which the force per contraction is increased compared to free wheel running, induced a more robust mTORC1 activation in both SOL and PLT. In this work, we present an easy-to-use, low stress, and time-efficient model to study mTORC1 signaling after resistance training in mice, which can be used in future studies to pin-down the molecular signaling events that determine muscle growth upon muscular contractions.

\section{DATA AVAILABILITY STATEMENT}

The datasets generated for this study are available on request to the corresponding author.

\section{ETHICS STATEMENT}

All animal procedures were approved by the Veterinary office of the Canton of Zürich (license nr. ZH254-16).

\section{AUTHOR CONTRIBUTIONS}

GD'H and AP designed the study, wrote the manuscript and performed all the experiments and data analysis. EM helped performing the experiments and edited the manuscript. OB-N helped in drafting and revising the manuscript. KB designed the study and helped drafting and revising the manuscript.

\section{FUNDING}

Internal funding from ETH Zurich.

\section{ACKNOWLEDGMENTS}

We would like to thank Nicola Bundschuh for her support and technical assistance in the lab. 


\section{REFERENCES}

Abe, T., Kojima, K., and Kearns, C. F. (2003). Whole body muscle hypertrophy from resistance training: distribution and total mass. Br. J. Sport. Med. 37, 543-545. doi: 10.1136/bjsm.37.6.543

Ashton-Miller, J. A., He, Y., Kadhiresan, V. A., McCubbrey, D. A., and Faulkner, J. A. (1992). An apparatus to measure in vivo biomechanical behavior of dorsiand plantarflexors of mouse ankle. J. Appl. Physiol. 72, 1205-1211. doi: 10.1152/jappl.1992.72.3.1205

Baar, K., and Esser, K. (1999). Phosphorylation of p $70^{\text {s6k }}$ correlates with increased skeletal muscle mass following resistance exercise. Am. J. Physiol. Physiol. 276, C120-C127. doi: 10.1152/ajpcell.1999.276.1.C120

Bhasin, S., Storer, T. W., Berman, N., Callegari, C., Clevenger, B., Phillips, J., et al. (1996). The effects of Supraphysiologic doses of testosterone on muscle size and strength in normal men. N. Engl. J. Med. 335, 1-7. doi: 10.1056/ NEJM199607043350101

Bodine, S. C., Stitt, T. N., Gonzalez, M., Kline, W. O., Stover, G. L., Bauerlein, R., et al. (2001). Akt/mTOR pathway is a crucial regulator of skeletal muscle hypertrophy and can prevent muscle atrophy in vivo. Nat. Cell Biol. 3, 1014-1019. doi: 10.1038/ncb1101-1014

Brook, M. S., Wilkinson, D. J., Mitchell, W. K., Lund, J. N., Szewczyk, N. J., Greenhaff, P. L., et al. (2015). Skeletal muscle hypertrophy adaptations predominate in the early stages of resistance exercise training, matching deuterium oxidederived measures of muscle protein synthesis and mechanistic target of rapamycin complex 1 signaling. FASEB J. 29, 4485-4496. doi: 10.1096/fj.15-273755

Burd, N. A., Holwerda, A. M., Selby, K. C., West, D. W. D., Staples, A. W., Cain, N. E., et al. (2010). Resistance exercise volume affects myofibrillar protein synthesis and anabolic signalling molecule phosphorylation in young men. J. Physiol. 588, 3119-3130. doi: 10.1113/jphysiol.2010.192856

Call, J. A., McKeehen, J. N., Novotny, S. A., and Lowe, D. A. (2010). Progressive resistance voluntary wheel running in the $\mathrm{mdx}$ mouse. Muscle Nerve 42 , 871-880. doi: 10.1002/mus.21764

Cholewa, J., Guimarães-Ferreira, L., da Silva Teixeira, T., Naimo, M. A., Zhi, X., de Sá, R. B. D. P., et al. (2014). Basic models modeling resistance training: an update for basic scientists interested in study skeletal muscle hypertrophy. J. Cell. Physiol. 229, 1148-1156. doi: 10.1002/jcp.24542

De Bono, J. P., Adlam, D., Paterson, D. J., and Channon, K. M. (2005). Novel quantitative phenotypes of exercise training in mouse models. Am. J. Physiol. Integr. Comp. Physiol.. 290, R926-R934. doi: 10.1152/ajpregu.00694.2005

Dreyer, H. C., Fujita, S., Glynn, E. L., Drummond, M. J., Volpi, E., and Rasmussen, B. B. (2010). Resistance exercise increases leg muscle protein synthesis and mTOR signalling independent of sex. Acta Physiol. 199, 71-81. doi: 10.1111/j.1748-1716.2010.02074.x

Drummond, M. J., Fry, C. S., Glynn, E. L., Dreyer, H. C., Dhanani, S., Timmerman, K. L., et al. (2009). Rapamycin administration in humans blocks the contraction-induced increase in skeletal muscle protein synthesis. J. Physiol. 587, 1535-1546. doi: 10.1113/jphysiol.2008.163816

Figueiredo, V. C., Caldow, M. K., Massie, V., Markworth, J. F., Cameron-Smith, D., and Blazevich, A. J. (2015). Ribosome biogenesis adaptation in resistance training-induced human skeletal muscle hypertrophy. Am. J. Physiol. Metab. 309, E72-E83. doi: 10.1152/ajpendo.00050.2015

Goldberg, A. L. (1968). Protein synthesis during work-induced growth of skeletal muscle. J. Cell Biol. 36, 653-658.

Goldspink, G. (1999). Changes in muscle mass and phenotype and the expression of autocrine and systemic growth factors by muscle in response to stretch and overload. J. Anat. 194, 323-334. doi: 10.1046/J.1469-7580.1999.19430323.X

Goodman, C. A., Frey, J. W., Mabrey, D. M., Jacobs, B. L., Lincoln, H. C., You, J. S., et al. (2011). The role of skeletal muscle mTOR in the regulation of mechanical load-induced growth. J. Physiol. 589, 5485-5501. doi: 10.1113/ jphysiol.2011.218255

Henneman, E., Somjen, G., and Carpentier, D. (1965). Functional significance of cell size in spinal motor neurons. J. Neurophysiol. 28, 560-580. doi: 10.1152/ jn.1965.28.3.560

Holland, A. M., Kephart, W. C., Mumford, P. W., Mobley, C. B., Lowery, R. P., Shake, J. J., et al. (2016). Effects of a ketogenic diet on adipose tissue, liver, and serum biomarkers in sedentary rats and rats that exercised via resisted voluntary wheel running. Am. J. Physiol. Integr. Comp. Physiol. 311, R337-R351. doi: 10.1152 /ajpregu.00156.2016
Huang, K., and Fingar, D. C. (2014). Growing knowledge of the mTOR signaling network. Semin. Cell Dev. Biol. 36, 79-90. doi: 10.1016/J.SEMCDB.2014.09.011

Janssen, I., Heymsfield, S. B., and Ross, R. (2002). Low relative skeletal muscle mass (sarcopenia) in older persons is associated with functional impairment and physical disability. J. Am. Geriatr. Soc. 50, 889-896. doi: 10.1046/j.15325415.2002.50216.x

Konhilas, J. P., Widegren, U., Allen, D. L., Paul, A. C., Cleary, A., and Leinwand, L. A. (2005). Loaded wheel running and muscle adaptation in the mouse. Am. J. Physiol. Circ. Physiol. 289, H455-H465. doi: 10.1152/ ajpheart.00085.2005

Kubica, N., Bolster, D. E., Farrell, P. A., Kimball, S. E., and Jefferson, L. S. (2005). Resistance exercise increases muscle protein synthesis and translation of eukaryotic initiation factor $2 \mathrm{~B} \varepsilon$ mRNA in a mammalian target of rapamycindependent manner. J. Biol. Chem. 280, 7570-7580. doi: 10.1074/jbc.M413732200

Legerlotz, K., Elliott, B., Guillemin, B., and Smith, H. K. (2008). Voluntary resistance running wheel activity pattern and skeletal muscle growth in rats. Exp. Physiol. 93, 754-762. doi: 10.1113/expphysiol.2007.041244

Lessard, S. J., MacDonald, T. L., Pathak, P., Han, M. S., Coffey, V. G., Edge, J., et al. (2018). JNK regulates muscle remodeling via myostatin/SMAD inhibition. Nat. Commun. 9:3030. doi: 10.1038/s41467-018-05439-3

Mahajan, P. B. (1994). Modulation of transcription of rRNA genes by rapamycin. Int. J. Immunopharmacol. 16, 711-721.

Mobley, C. B., Holland, A. M., Kephart, W. C., Mumford, P. W., Lowery, R. P., Kavazis, A. N., et al. (2018). Progressive resistance-loaded voluntary wheel running increases hypertrophy and differentially affects muscle protein synthesis, ribosome biogenesis, and proteolytic markers in rat muscle. J. Anim. Physiol. Anim. Nutr. 102, 317-329. doi: 10.1111/jpn.12691

O’Neil, T. K., Duffy, L. R., Frey, J. W., and Hornberger, T. A. (2009). The role of phosphoinositide 3-kinase and phosphatidic acid in the regulation of mammalian target of rapamycin following eccentric contractions. J. Physiol. 587, 3691-3701. doi: 10.1113/jphysiol.2009.173609

Ogasawara, R., and Suginohara, T. (2018). Rapamycin-insensitive mechanistic target of rapamycin regulates basal and resistance exercise-induced muscle protein synthesis. FASEB J. 32, 5824-5834. doi: 10.1096/f.201701422R

Philp, A., Schenk, S., Perez-Schindler, J., Hamilton, D. L., Breen, L., Laverone, E., et al. (2015). Rapamycin does not prevent increases in myofibrillar or mitochondrial protein synthesis following endurance exercise. J. Physiol. 593, 4275-4284. doi: 10.1113/JP271219

Pyka, G., Lindenberger, E., Charette, S., and Marcus, R. (1994). Muscle strength and fiber adaptations to a year-long resistance training program in elderly men and women. J. Gerontol. 49, M22-M27. doi: 10.1093/ geronj/49.1.M22

Rahnert, J. A., and Burkholder, T. J. (2013). High-frequency electrical stimulation reveals a p38-mTOR signaling module correlated with force-time integral. J. Exp. Biol. 216, 2619-2631. doi: 10.1242/jeb.080705

Rivero-Gutiérrez, B., Anzola, A., Martínez-Augustin, O., and De Medina, F. S. (2014). Stain-free detection as loading control alternative to Ponceau and housekeeping protein immunodetection in Western blotting. Anal. Biochem. 467, 1-3. doi: 10.1016/j.ab.2014.08.027

Roach, G. C., Edke, M., and Griffin, T. M. (2012). A novel mouse running wheel that senses individual limb forces: biomechanical validation and in vivo testing. J. Appl. Physiol. 113, 627-635. doi: 10.1152/japplphysiol.00272.2012

Roemers, P., Mazzola, P. N., De Deyn, P. P., Bossers, W. J., van Heuvelen, M. J., and van der Zee, E. A. (2017). Burrowing as a novel voluntary strength training method for mice: a comparison of various voluntary strength or resistance exercise methods. J. Neurosci. Methods 300, 112-126. doi: 10.1016/j. jneumeth.2017.05.027

Scribbans, T. D., Ma, J. K., Edgett, B. A., Vorobej, K. A., Mitchell, A. S., Zelt, J. G. E., et al. (2014). Resveratrol supplementation does not augment performance adaptations or fibre-type-specific responses to high-intensity interval training in humans. Appl. Physiol. Nutr. Metab. 39, 1305-1313. doi: 10.1139/apnm-2014-0070

Sher, J., and Cardasis, C. (1976). Skeletal muscle fiber types in the adult mouse. Acta Neurol. Scand. 54, 45-56. doi: 10.1111/j.1600-0404.1976.tb07619.x

Soffe, Z., Radley-Crabb, H. G., McMahon, C., Grounds, M. D., and Shavlakadze, T. (2016). Effects of loaded voluntary wheel exercise on performance and muscle hypertrophy in young and old male C57Bl/6J mice. Scand. J. Med. Sci. Sports 26, 172-188. doi: 10.1111/sms.12416 
Staron, R. S., Karapondo, D. L., Kraemer, W. J., Fry, A. C., Gordon, S. E., Falkel, J. E., et al. (1994). Skeletal muscle adaptations during early phase of heavy-resistance training in men and women. J. Appl. Physiol. 76, 1247-1255. doi: 10.1152/jappl.1994.76.3.1247

Szulc, P., Beck, T. J., Marchand, F., and Delmas, P. D. (2004). Low skeletal muscle mass is associated with poor structural parameters of bone and impaired balance in elderly men-the MINOS study. J. Bone Miner. Res. 20, 721-729. doi: 10.1359/JBMR.041230

Szulc, P., Munoz, F., Marchand, F., Chapurlat, R., and Delmas, P. D. (2010). Rapid loss of appendicular skeletal muscle mass is associated with higher all-cause mortality in older men: the prospective MINOS study. Am. J. Clin. Nutr. 91, 1227-1236. doi: 10.3945/ajcn.2009.28256

Thoreen, C. C., Chantranupong, L., Keys, H. R., Wang, T., Gray, N. S., and Sabatini, D. M. (2012). A unifying model for mTORC1-mediated regulation of mRNA translation. Nature 485, 109-113. doi: 10.1038/nature11083

Verdijk, L. B., Gleeson, B. G., Jonkers, R. A. M., Meijer, K., Savelberg, H. H. C. M., Dendale, P., et al. (2009). Skeletal muscle hypertrophy following resistance training is accompanied by a fiber type-specific increase in satellite cell content in elderly men. J. Gerontol. Ser. A Biol. Sci. Med. Sci. 64A, 332-339. doi: $10.1093 /$ gerona/gln050
White, Z., Terrill, J., White, R. B., McMahon, C., Sheard, P., Grounds, M. D., et al. (2016). Voluntary resistance wheel exercise from mid-life prevents sarcopenia and increases markers of mitochondrial function and autophagy in muscles of old male and female C57BL/6J mice. Skelet. Muscle 6, 1-21. doi: 10.1186/s13395-016-0117-3

Wolfson, R. L., and Sabatini, D. M. (2017). The dawn of the age of amino acid sensors for the mTORC1 pathway. Cell Metab. 26, 301-309. doi: 10.1016/j. cmet.2017.07.001

Conflict of Interest: The authors declare that the research was conducted in the absence of any commercial or financial relationships that could be construed as a potential conflict of interest.

Copyright (c) 2019 D'Hulst, Palmer, Masschelein, Bar-Nur and De Bock. This is an open-access article distributed under the terms of the Creative Commons Attribution License (CC BY). The use, distribution or reproduction in other forums is permitted, provided the original author(s) and the copyright owner(s) are credited and that the original publication in this journal is cited, in accordance with accepted academic practice. No use, distribution or reproduction is permitted which does not comply with these terms. 